\title{
Cellular mechanisms of white matter regeneration in an adult dysmyelinated rat model
}

\author{
Jacek M. Kwiecien \\ Department of Pathology and Molecular Medicine, Michael G. DeGroote School of Medicine, McMaster University, Hamilton, Ontario, \\ Canada
}

\begin{abstract}
Cellular mechanisms of regeneration after the white matter injury are difficult to study because of severe, inflammatory response to massively damaged myelin. Myelin-lacking CNS of the adult Long Evans Shaker (LES) rat supplies a model where neuroregeneration can be studied conveniently. The crush site in the dorsal spinal column in LES rats implanted with the normal rat choroid plexus was studied under the light and electron microscopy at 5 time points 3-56 days post-op. While the crush injury in normal rats resulted in severe inflammation active beyond 8 weeks, the same injury in LES rats resulted in a brief inflammation that resolved before day 7 post-op. In a clear fluid-filled crush cavity, ependymal cells from the implanted choroid plexus encased multiple regenerating axons, apparently guided them across the crush cavity and participated in establishing of a zone of neuroregeneration, morphologically similar to the white matter, at the interface of the crush cavity and the surrounding tissue of the spinal cord. Axons that were not encased by implanted cells failed to cross the crush cavity and persisted as markedly swollen end bulbs filled with organelles. At 8 weeks post-op, a large proportion of axons in the zone of neuroregeneration became myelinated by Schwann cells, likely originating from dorsal nerve roots or by oligodendrocytes that formed thin sheaths with a major dense line and likely originated from the implanted choroid plexus. The LES rat can serve as a convenient model to study mechanisms of neuroregeneration including axonal regeneration in the adult CNS injury.
\end{abstract}

Key words: spinal cord injury, axonal regeneration, choroid plexus implant, inhibition of leukomyelitis, transdifferentiation of ependymal cells to oligodendrocytes, zone of neuroregeneration.

\section{Introduction}

Cellular mechanisms of neuroregeneration including axonal regeneration are difficult to study in vivo in the adult central nervous system (CNS). Traumatic injury to the white matter results in widespread myelin damage and is rapidly followed by severe, macrophage-rich inflammatory infiltration that perpetuates myelin damage and thus attracts more mac- rophages $[5,22]$. This vicious cycle of events remains severe over a period of several months and results in expansion of inflammation and destruction of the CNS tissue. In myelinated animal models therefore, studies on neuroregeneration have been seriously hampered by inflammatory response to damaged myelin rendering research progress towards meaningful, regenerative therapies for patients with spi- 
nal cord injury and brain trauma, difficult. Since the spinal cord injury, brain trauma and stroke result in a loss of neural tissue and transection of axons that are related to a loss of the neurological function, a variety of strategies have been pursued to restore the lost CNS tissue and reduce the severity of neurological deficits $[22,25]$ with inconclusive results.

In this study, cellular mechanisms of regeneration were analyzed in the acute crush injury of the dorsal column of the spinal cord of mature Long Evans Shaker (LES) rats implanted with rat choroid plexus. The LES rat is a mutant of myelin basic protein [18] characterized by a complete lack of CNS myelin $[10,13]$ and the longevity similar to that of a normal laboratory rat [10]. Despite its inherent fragility related to its propensity to seizures and severe convulsions [12], the adult LES rats can survive neurosurgeries for a number of weeks in good health [11, 20] making it an attractive model to study regenerative processes in the CNS.

Although ependymal cells lining the ventricular system in the brain and the central canal in the spinal cord and the rat filum terminale [11] are highly differentiated, they are considered neural stem cells able to proliferate and supply neural progenitors in the intact CNS $[8,26]$. The ependymal cells have been shown to react to the spinal cord injury or brain trauma by proliferating, migrating towards the lesion site and differentiating into neural progenitors and glial cells $[4,15,17,24,27]$ and by actively participating in supporting axonal regeneration following the crush of the filum terminale of a novel rat model of vivo CNS injury [11]. The choroid plexus is a specialized organ in the brain ventricles, which constantly secretes the cerebrospinal fluid (CSF), considered an interstitial fluid of the CNS, and can participate in repair reaction to the trauma [2]. This exquisitely folded, cauliflower-like organ is supported by delicate fibrovascular stroma and is covered by a single layer of low columnar epithelium that is contiguous with ependymal lining of the ventricular system in the brain and the central canal in the spinal cord [19] and in the filum terminale of the rat [11]. The epithelial cells of the choroid plexus originate from ependyma [2] and are considered modified ependymal cells $[1,6,7,9]$. The choroid plexus ependymal cells have been shown to contribute neural progenitor cells [7] and to closely associate with axons when implanted into the spinal cord injury [6]. In the spinal cord injury site they have been shown to differentiate primarily into astrocytes [9].

Lack of myelin in the LES rat CNS allows for the naked axons to retain plasticity and to sprout spontaneously in the intact rats throughout their lifespan [10]. Sprouting activity was shown to be markedly augmented in the sacral spinal cord in response to the crush injury of the filum terminale, $5 \mathrm{~mm}$ distal, indicating responsiveness of the axonal plasticity to the CNS tissue damage [11]. Cut axons in the LES rat spinal cord do not regenerate on their own however, presumably because they do not cross the fluid-filled cavity forming rapidly after the crush injury [16]. Implantation of a suitable cell population, the rat choroid plexus that would support axonal regeneration at the site of the crush injury was attempted in this study and the cellular events participating in axonal regeneration analyzed under the light and electron microscopy.

\section{Material and methods}

\section{Animals}

The Long Evans Shaker (LES) rats are mutants of myelin basic protein, do not form proper CNS myelin $[13,18]$ and are completely myelin-free in adult life [10]. Lack of myelin in the CNS of LES rats allows for studies of cellular events after implantation of intact or injured white matter. Also, axonal plasticity and regeneration can be conveniently studied in the dysmyelinated CNS of the LES rats.

The LES and control LE rats, phenotypically normal littermates of LES, were housed specific pathogen-free in a conventional rodent facility, Central Animal Facility, McMaster University, and given Rodent chow (Lab Diets, PMI Feeds, Inc., St. Louis, MO) and tap water ad libitum. The raising of the animals and the experimental procedures were approved by the Animal Research Ethics Board, McMaster University, and conducted according to guidelines by the Canadian Council of Animal Care. The LES rats, 15 weeks or older, 4 per survival period group $(n=40)$ were used for experimental neurosurgeries. Adult phenotypically normal littermates were used in this study as controls.

\section{Dorsal spinal column crush}

The spinal crush injury was limited to the dorsal spinal column for two reasons: (1) the injury did not result in paralysis of the urinary bladder that would lead to the retention of urine, hemorrhagic 
cystitis and the need to euthanize the LES rat at the humane endpoint [12]; (2) crushed central axons of dorsal root ganglion neurons in ascending sensory pathways of the fasciculus cuneatus and gracilis can be visualized and studied by an appropriate tracer injected into the sciatic nerves $[14,16]$.

The choroid plexus was used for implantation of the acute spinal cord injury in this study following a previous observation that ependymal cells actively supported axonal regeneration following the filum terminale crush in rats [11]. In the cited study, immature cells interpreted as ependymal cells dissociated from the central canal, migrated within the crush injury and encased multiple axons that were then apparently directed towards the central canal of the filum terminale caudal to the crush. There ependymal cells formed channels with their basal processes where axonal regeneration occurred. Axonal regeneration was exclusive to the channels formed by ependymal cells and did not occur in the absence of ependymal cells [11]. Epithelial cells in the choroid plexus are considered modified ependymal cells $[1,6,7,9]$.

Adult, > 15-week-old LES rats were induced and maintained under isoflurane anaesthesia and skin on the dorsum prepared surgically. Dorsal laminectomy was performed on the T8 vertebrae, the spinal cord exposed and dura mater cut sagittally to expose the dorsal spinal cord, at mid-thoracic region. The dorsal column was crushed with fine jeweller's forceps for 10 seconds, choroid plexus harvested from the adult LE-control rat was implanted into the crush lesion and a spinal muscle fascia was placed over the lesion to seal it. The LES rats that had dorsal column crushed but not implanted served as controls. The neurosurgeries on LE-control rats were performed in a similar fashion. The spinal muscles were closed with an absorbable suture, the skin was closed with stainless steel staples and the surgery rats were treated with $5 \mathrm{~mL}$ saline subcutaneous and 0.2-0.3 mL Anafen ${ }^{\circledR}$ (ketoprofen, $10 \mathrm{mg} / \mathrm{mL}$, Merial) subcutaneous prior to awaking from anaesthesia. A total of 20 LES rats implanted and 20 LES rats not implanted survived in good health to their respective time points, 3-56 days post-op and were used in this study.

\section{CNS tissue collection and analysis}

The whole body perfusion and fixation was performed with Karnowski's fixative [11]. Semithin cross sections of the spinal cord with the crush lesion were stained with toluidine blue and examined under a Nikon Eclipse 50i light microscope. Silver gray ultrathin sections from Epon-embedded portions of the optic nerve and spinal cord were mounted on Formvar coated copper grids, stained with uranyl acetate and lead citrate and examined under a Jeol 1200EX Biosystem transmission electron microscope [11].

\section{Results}

Analysis of the site of the crush injury in the dorsal spinal column in mature LES rats implanted with rat choroid plexus was performed with light and electron microscopy in this study. Remarkable histological and electron microscopic changes are shown in Figs. 1-8. The white matter crush injury resulted in dramatically different pathology in normal LE rats than in LES rats. Brief inflammatory response to the crush injury in the LES rats allowed for morphological analysis of cellular events participating in axonal regeneration and formation of new white matter-type tissue. Severe, protracted, expansive and destructive inflammatory reaction to damaged myelin in LE control rats obliterated implanted choroid plexus and did not allow for observation of any cellular events interpreted as neuroregeneration.

\section{Inflammation}

The crush injury in the dorsal column of a normally myelinated spinal cord of LE-control rats resulted in a severe, macrophage-rich inflammatory response that was progressive and still active at 56 days after the injury (Fig. 1). Macrophages invariably encompassed toluidine blue-positive debris interpreted as phagocytized myelin. In previous experiments, cells or synthetic materials implanted into the spinal crush injury of LE-control rats were invariably obliterated by a severe, macrophage-rich inflammatory response, thus rendering analysis of cellular interactions with the implanted CNS difficult or impossible [Kwiecien, unpublished]. Since the CNS of the LES rat is completely devoid of myelin $[10,13]$, inflammatory reaction against damaged myelin does not take place in this rat model of the spinal cord injury. The inflammatory response in the spinal cord of the LES rats that were not implanted included haemorrhages and macrophage infiltration 

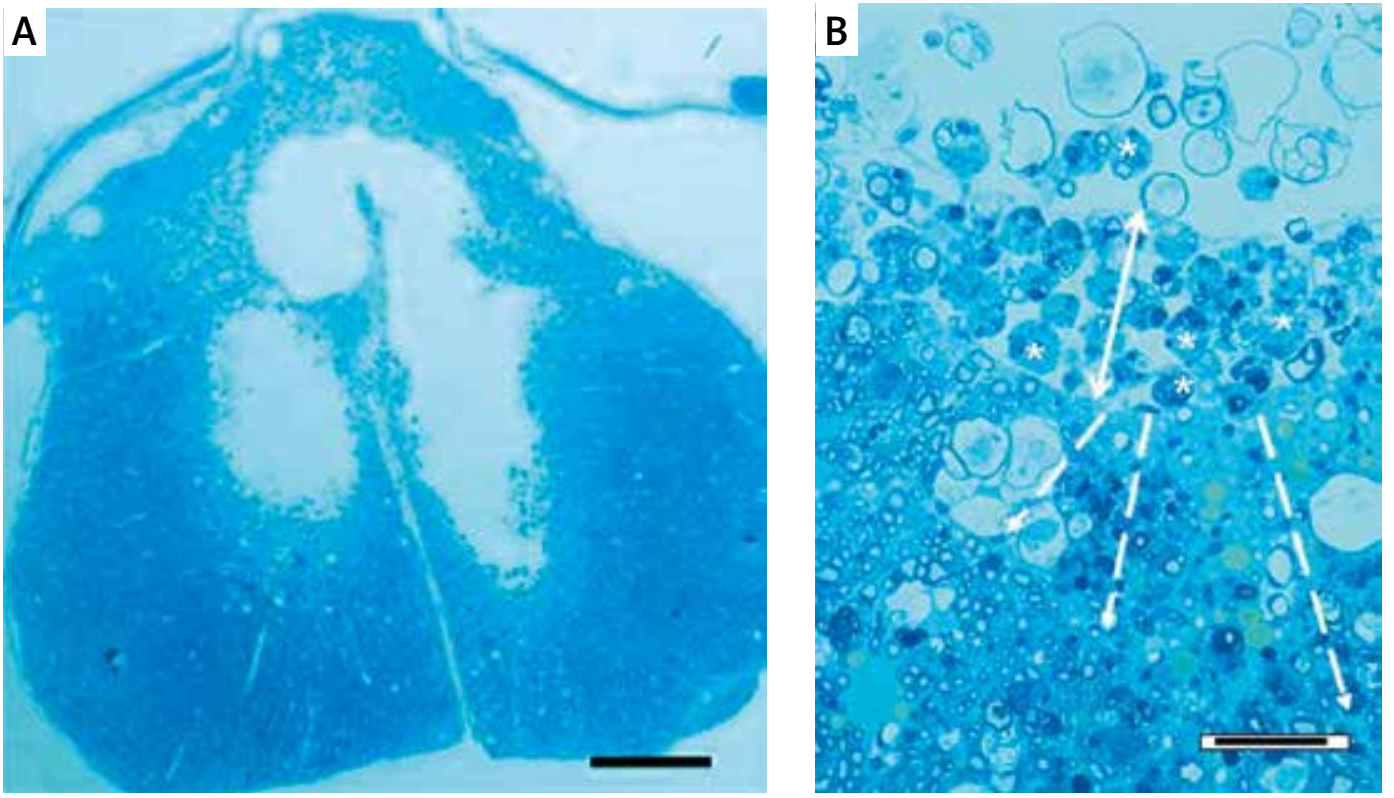

Fig. 1. Dorsal spinal column crush in the control, normally myelinated Long Evans rat, 56 days post-op. (A) The dorsal column and adjacent areas of white and gray matter are obliterated and replaced by a fluid-filled cavity. Toluidine blue (TB), bar $500 \mu \mathrm{m}$. (B) There is marked, several cell deep infiltration at the interface of the cavity and the surrounding spinal cord tissue (solid double-headed arrow) by large, round cells rich in intracytoplasmic, dark blue debris (asterisks). These cells are interpreted as macrophages and dark blue debris as phagocytized myelin debris. In the adjacent tissue of white matter there is a large area of diffuse demyelination (interrupted arrows), adjacent to preserved myelin sheaths, bottom left. TB, bar $50 \mu \mathrm{m}$.

among the cellular debris in the crush lesion at day 3 post-op that cleared completely before day 7 resulting in a clear fluid-filled crush cavity. In the implanted LES rats, of the large amount of the choroid plexus implanted into the crush injury, only a small proportion of implanted ependymal cells survived in the crush cavity. At 3 days post-crush (post-op), most of cells in the crush cavity were red blood cells and macrophages (Fig. 2). Although blood vessels and small sheets of columnar epithelium were evident, most of the implanted tissue of the morphologically recognizable choroid plexus was not found in the crush cavity at 3 days post-op. In the tissue of the spinal cord surrounding the crush injury there were many large swollen axons packed with microtubules and other organelles (Fig. 3). Starting at 7 days post-op, the crush cavity became devoid of inflammatory cells (Fig. 4) except for an occasional macrophage (Fig. 4D). This rapid clearing of inflammatory cells allowed for unencumbered analysis of cellular mechanisms of neuroregeneration taking place in the crush cavity and in the surrounding tissue of the spinal cord.

\section{Axonal regeneration}

In the crush injury site axons were not observed in LES rats not implanted with choroid plexus and in LE-control rats. In LES rats, axons in the spinal cord tissue surrounding the crush cavity are unmyelinated and separated from each other by severe, diffuse astrogliosis $[10,13]$. In areas adjacent to the margin of the crush cavity, and also at the interface of the crush cavity and surrounding CNS tissue, there were numerous individual or clusters of markedly swollen axons packed with neurofilaments arranged in multidirectional streams admixed with neurotubules and numerous mitochondria and vesicles (Figs. 3B, 7B).

At 3 days post-op, throughout the crush cavity containing fluid with scattered red blood cells, cellular debris and macrophages, there were small fragments of columnar epithelium interpreted as implanted rat choroid plexus (Fig. 2). Some of the epithelial cells encased axonal profiles (Figs. 2B, C). At 7 days post-op, numerous axons were encased by cytoplasmic processes of adjacent dissociated 

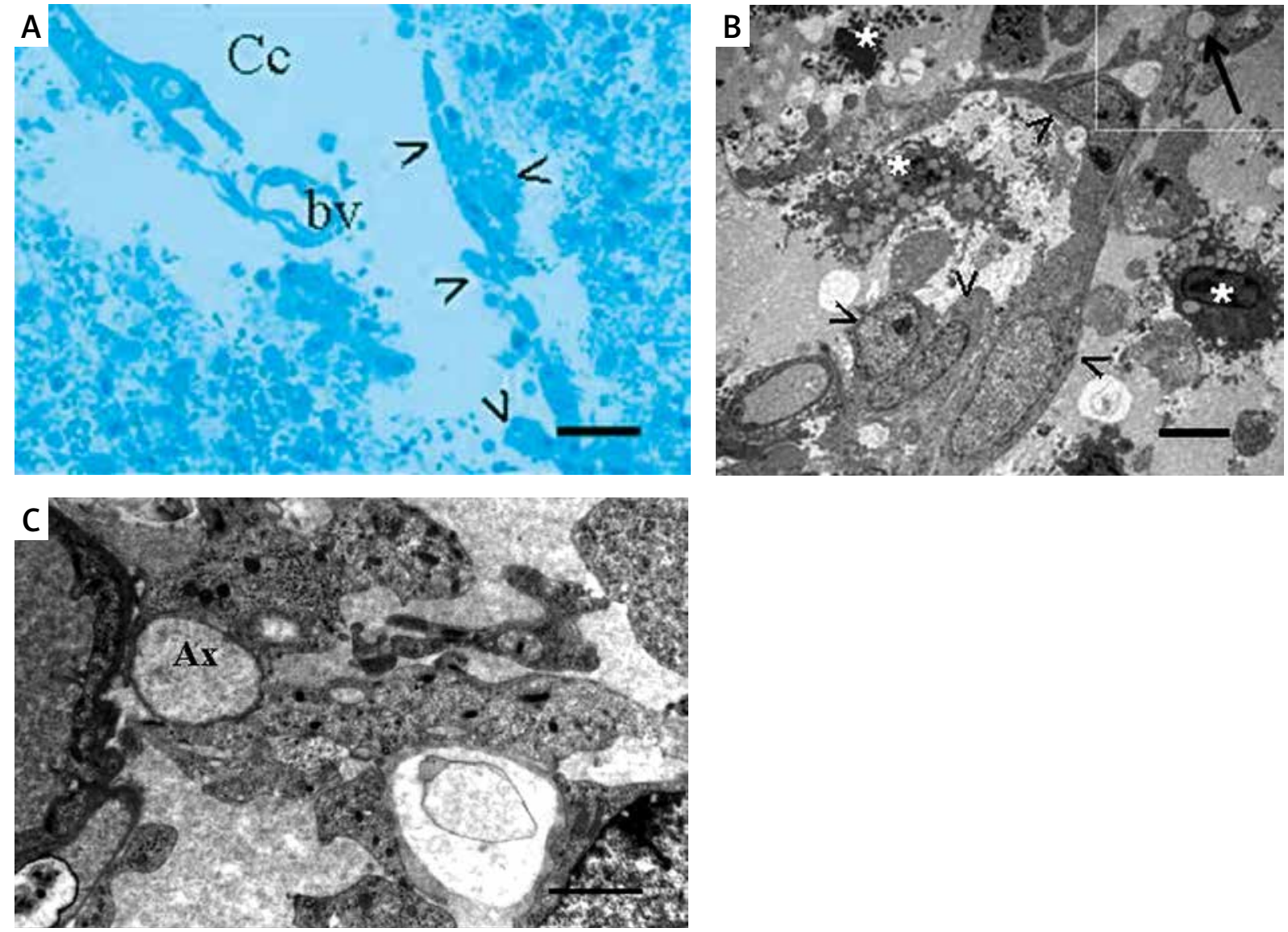

Fig. 2. Dorsal spinal column crush in the adult LES rat implanted with rat choroid plexus, 3 days post-op. (A) The crush cavity (Cc) contains clear fluid, presumably the cerebrospinal fluid (CSF) with blood vessels (bv) and sheets of epithelium (open arrowheads) interpreted as from implanted choroid plexus are surrounded by haemorrhages and scattered cells, some interpreted as macrophages. TB, bar $50 \mu \mathrm{m}$. (B) Transmission electron micrograph (TEM) from the crush cavity contains a small sheet of 4 columnar cells (open arrowheads) interpreted as part of implanted choroid plexus. A process of one of the epithelial cells encompasses one axon (arrow). There are macrophages with rich fillipodia (asterisks) in the surrounding CSF. Bar $10 \mu \mathrm{M}$. (C) Detail of B delineated in the white box. An axon (Ax) is wrapped by a cell process continuous with the epithelial cell in the right lower corner from the implanted choroid plexus. Bar $2 \mu \mathrm{M}$.

cells throughout the crush cavity that was filled with clear fluid and virtually devoid of cellular debris and phagocytic cells (Fig. 4). At 14-56 days post-op, axons and encasing cells were clustered in the zone of neuroregeneration at the interface of the crush cavity and the surrounding spinal cord tissue (Figs. 5, 6, 8) with a narrow fluid-filled cleft separating the zone of neuroregeneration from the spinal cord tissue (Figs. 5A, 6A, 8B). Normal axonal profiles not encased by adjacent cells were not found at the dissociated stage (first 7 days post-op) or in the zone of neuroregeneration (14-56 days post-op).

\section{Myelination of regenerating axons}

Although the wrapping of the cytoplasmic processes around all axons in the zone of neuroregeneration was observed starting at day 3 post-op, many axons remained partially wrapped (Figs. 4C, 8C, E). Numerous myelin sheaths were scattered in the zone of neuroregeneration and in the adjacent areas of the spinal cord tissue at 56 days post-op (Fig. 8) but not at earlier post-op intervals. A large proportion of thick myelin sheaths in the zone of regeneration were around large diameter axons. They were com- 

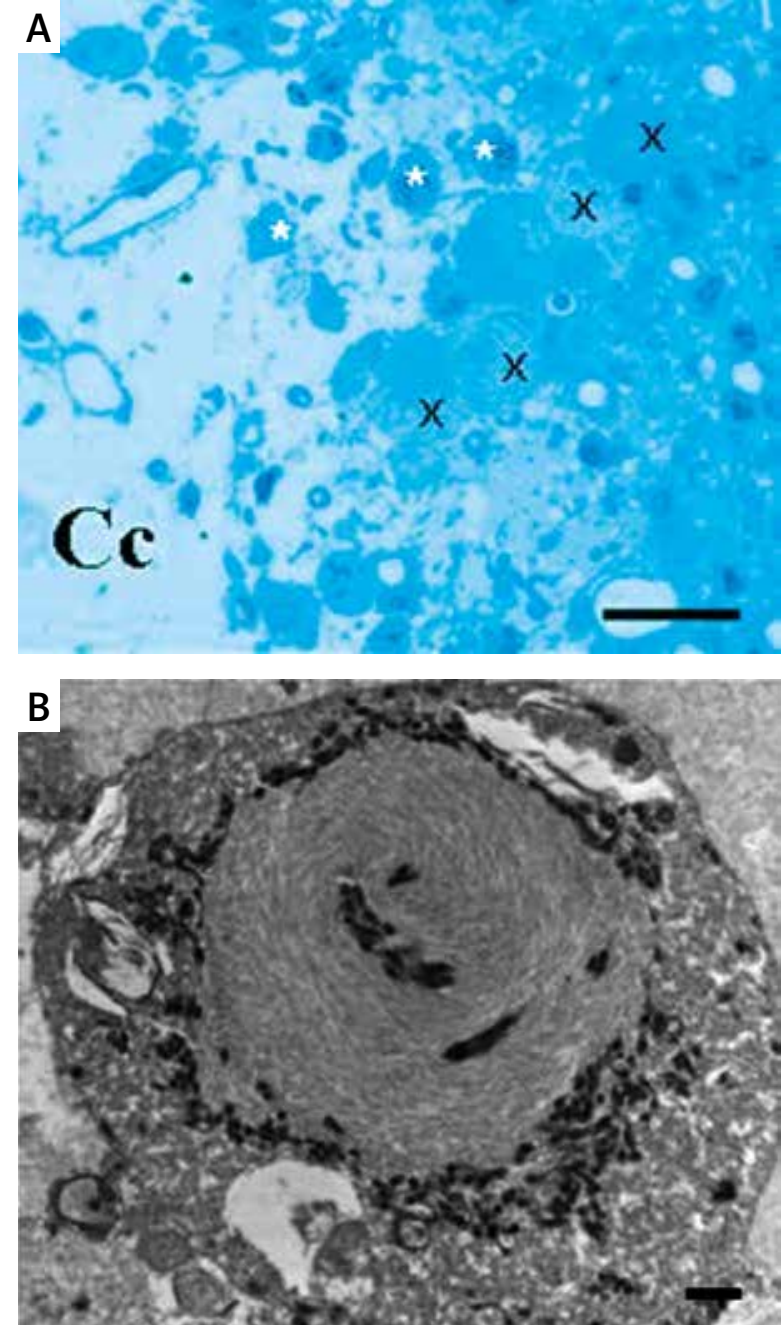

Fig. 3. Dorsal spinal column crush in the adult LES rat implanted with rat choroid plexus, 3 days post-op. (A) At the edge of the spinal cord tissue surrounding the crush cavity $(\mathrm{Cc})$ there are multiple adjacent swollen axons ( $x$ ) surrounded by haemorrhages and scattered macrophages (asterisks). TB, bar $50 \mu \mathrm{M}$. (B) TEM of a swollen axon with the centre occupied by tightly packed, whirling neurotubules surrounded by small dense bodies and cross-sections of microtubules apparently running along the axis of the axon. Bar $2 \mu \mathrm{M}$.

pacted, with a major dense line, surrounded by basal lamina and often adjacent to a Schwann cell (Figs. 8B, 8D). Remarkable Schwann cell type of myelination was also present in the areas of the spinal cord tissue adjacent to the crush cavity. Scattered in the zone of neuroregeneration there were large diameter axons surrounded by compacted, 2-3 lamellae-thick sheaths (Fig. 8E) that formed the major dense line (Fig. 8E insert). Cells adjacent to such sheaths had an irregular nucleus with chromatin clumped in the periphery and abundant cytoplasm rich in organelles including mitochondria, apparatus of Golgi, and microtubules but no basal lamina (Figs. 8B, 8E). Numerous axons in the zone of neuroregeneration remained unmyelinated (Figs. 8B-D).

\section{Implanted cells}

Implanted choroid plexus tissue included stromal blood vessels (Fig. 2A) and epithelial cells in small sheets of columnar epithelium at day 3 post-op (Figs. 2A,B). At 7-28 days post-op, blood vessels and epithelial sheets were not found, instead, dissociated, large cells, encasing multiple adjacent axons were found first throughout the crush cavity at day 7 postop (Fig. 4) and then, at days 14-28 post-op, in the zone of neuroregeneration (Figs. 5, 6). These cells were similar to ependymal cells of the central canal (Fig. 7A), had an oval or irregular, often subcleaved nucleus with large, irregular clumps of chromatin in the periphery and appeared to support axonal regeneration across the fluid-filled crush cavity. The cytoplasm was abundant, rich in organelles including mitochondria, vesicles, endoplasmic reticulum and ribosomes. In the crush cavity these cells invariably encased numerous adjacent axons (Figs. 4B, C). Similar cells without encased axons were not evident. Rare macrophages in the vicinity of regenerating axons did not encase them and had a banana-shaped nucleus and distinct numerous narrow fillipodia in the periphery (Fig. 4D). From day 14 post-op, together with encased axons, dissociated ependymal cells from implanted choroid plexus clustered together and formed a discontinuous 20-50 $\mu \mathrm{m}$ thick zone of neuroregeneration at the interface of the fluid-filled crush cavity and the surrounding tissue of the spinal cord (Figs. $5 \mathrm{~A}, 6 \mathrm{~A}, 8 \mathrm{~B})$ and the zone of neuroregeneration was delineated from the adjacent spinal cord tissue by a narrow, discontinuous cleft. Cells in the zone of neuroregeneration were separated from each other by spaces, presumably filled by clear fluid (Figs. 5, 6, 8). Although numerous myelinating Schwann cells were scattered in the zone of neuroregeneration at day 56 post-op, there were also large immature cells with an irregular nucleus with chromatin clumped in the periphery and abundant cytoplasm rich in organelles including mitochondria, apparatus of Golgi, and 

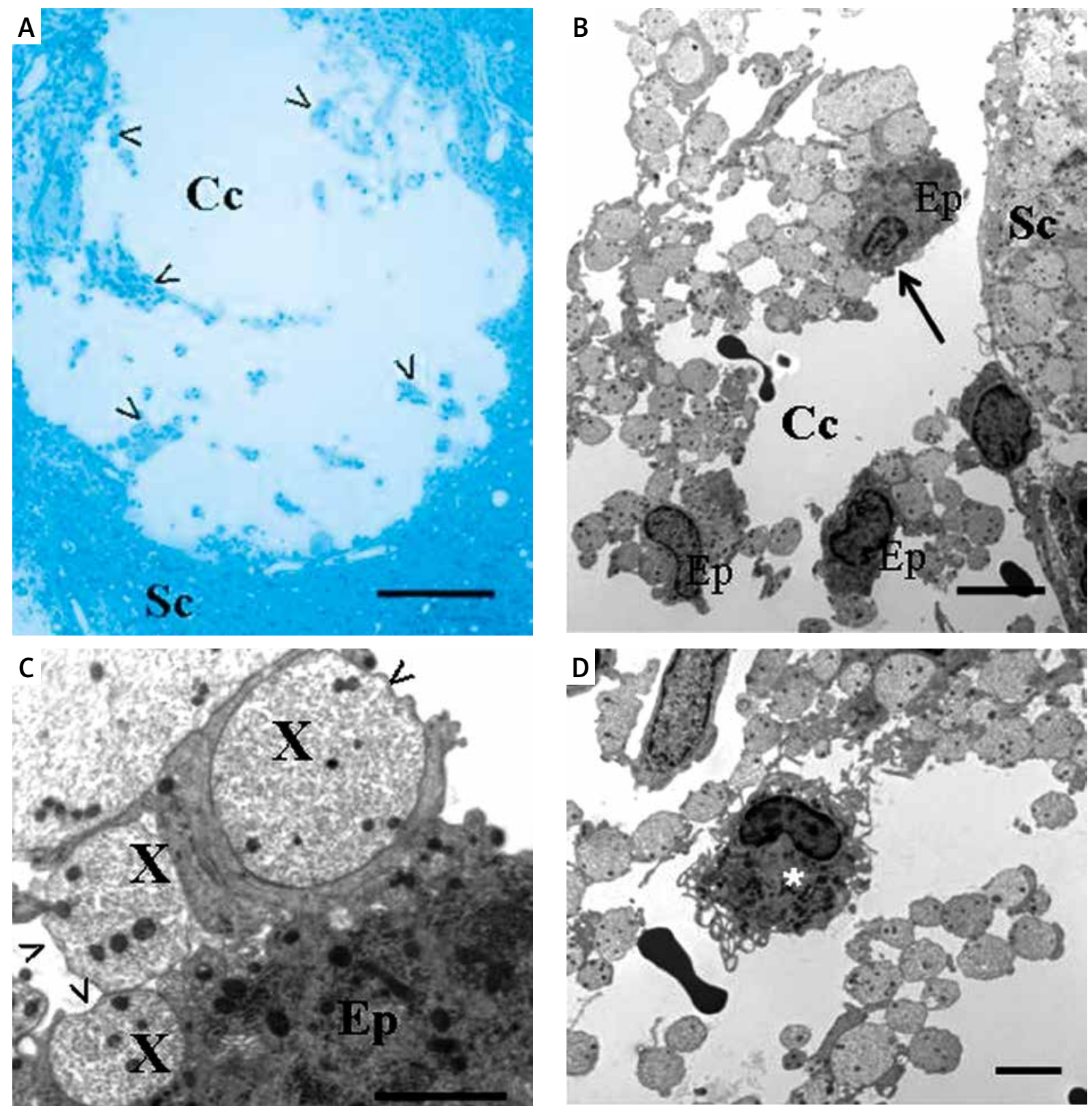

Fig. 4. Dorsal spinal column crush in the adult LES rat implanted with rat choroid plexus, 7 days post-op. (A) The crush cavity (Cc) contains clear fluid, presumably the CSF with delicate fronds of tissue (open arrowheads) scattered throughout is surrounded by spinal cord tissue (Sc). TB, bar $200 \mu \mathrm{m}$. (B) TEM with the area of the crush cavity $(\mathrm{Cc})$ adjacent to the surrounding spinal cord tissue $(\mathrm{Sc})$. There are 3 dissociated large cells interpreted as ependymal cells (Ep) from implanted choroid plexus that encase cross-sections of numerous axons with their processes. Bar $10 \mu \mathrm{m}$. (C) One ependymal cell (Ep) indicated by an arrow in (B) encases numerous adjacent axons with its cytoplasmic processes. Three axons adjacent to the cell body $(X)$ are incompletely wrapped around (open arrowhead) by the cytoplasmic processes. This cell is interpreted as an immature ependymal cell dissociated from the implanted choroid plexus epithelium and has an open, subcleaved nucleus and its large cytoplasm is rich in organelles including mitochondria, apparatuses of Golgi, ribosomes and the endoplasmic reticulum. Bar $2 \mu \mathrm{m}$. (D) In the crush cavity, there are scattered rare red blood cells and a rare macrophage (asterisk) with a cleaved nucleus, rich cytoplasm and numerous narrow fillipodia that do not encase surrounding axons. Bar $5 \mu \mathrm{m}$. 

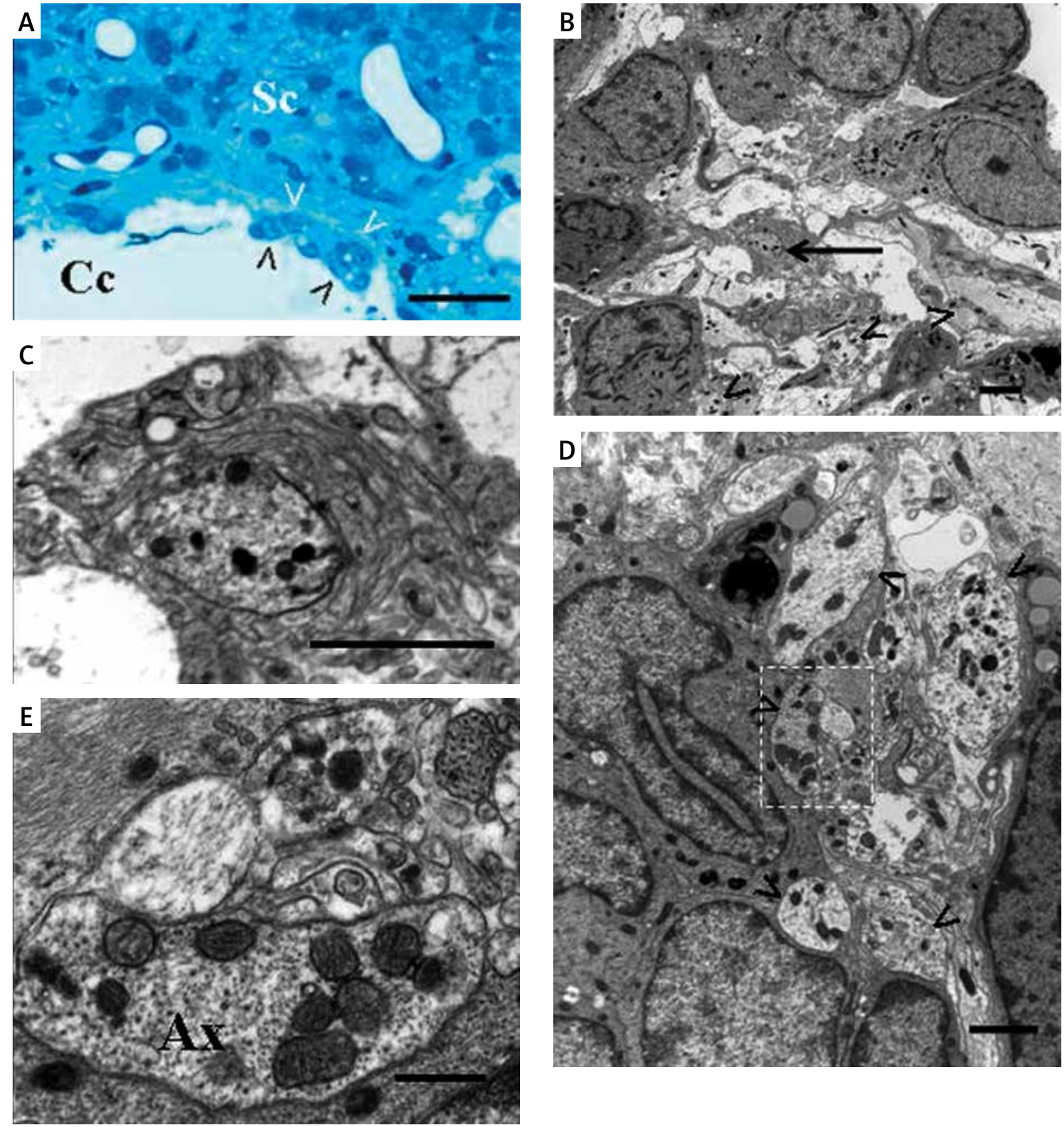

Fig. 5. Dorsal spinal column crush in the adult LES rat implanted with rat choroid plexus, 14 days post-op. (A) The crush cavity (CC) contains clear fluid, presumably the CSF with small clusters of cells (open arrowheads) at the interface of the crush cavity and separated by a narrow cleft (white arrowheads) from the surrounding spinal cord tissue (Sc). TB, bar $50 \mu \mathrm{m}$. (B) TEM of the cell cluster the margin of the crush cavity. A discontinuous ring of large cells surrounds an area rich in cell processes surrounded by clear fluid. The arrow and the open arrowheads indicate cross-sections of axons surrounded by processes from surrounding cells. Bar $5 \mu \mathrm{m}$. (C) Higher magnification of an area from B that includes the axon (arrow) surrounded by cellular processes. Bar $2 \mu \mathrm{m}$. (D) In a different area, a cluster of large cells at the interface of the crush cavity and the surrounding spinal cord tissue surround a cluster of large axons (arrowheads). Bar $5 \mu \mathrm{m}$. (E) Higher magnification of the area within the box in (D). A large axon contains mitochondria, microtubules and neurofilaments. Bar $500 \mathrm{~nm}$. 

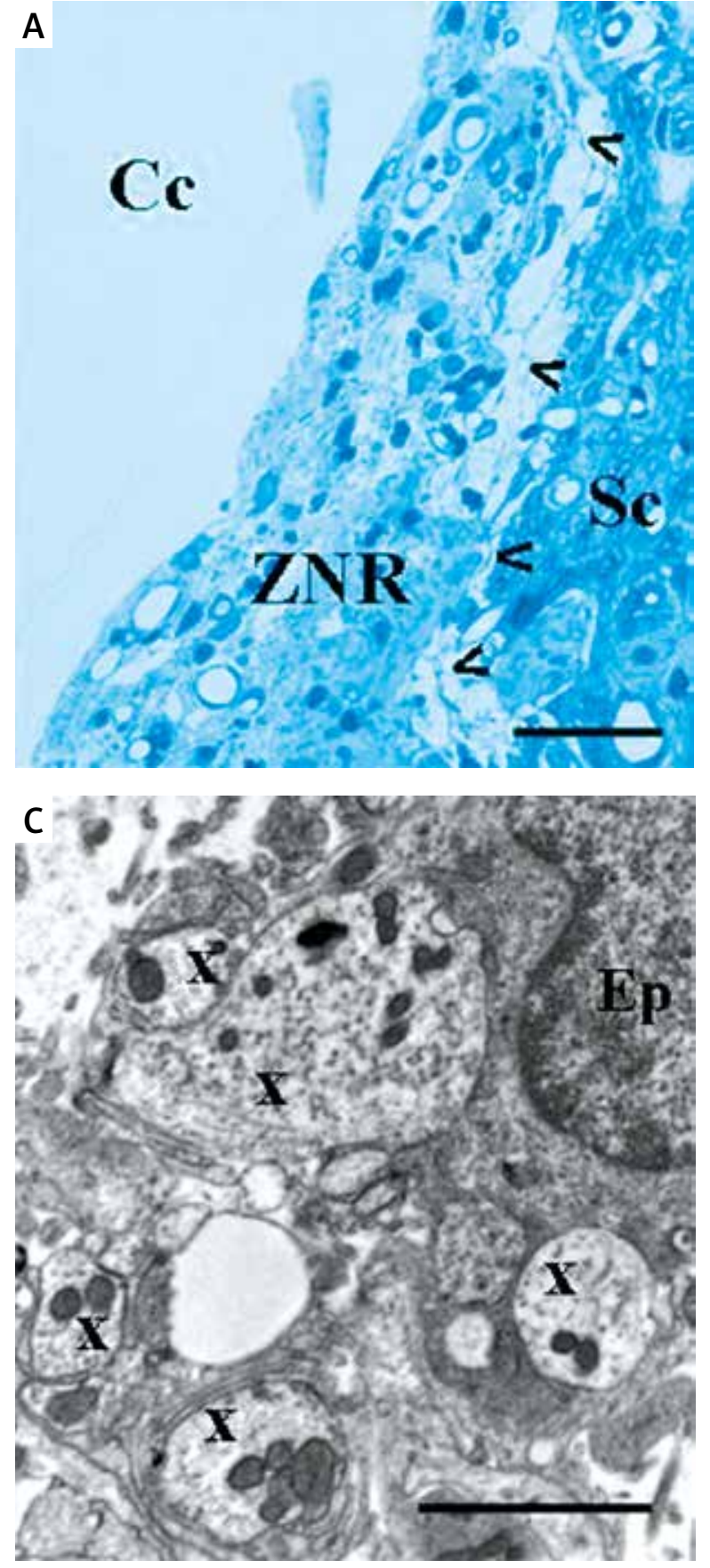

microtubules but no basal lamina (Figs. 8B, C, E). The latter cells sometimes were immediately adjacent to large axons with 2-3 lamellae-thick sheath (Fig. 8E) with a formed major dense line (Fig. 6E insert). Such cells were interpreted as oligodendrocytes [19] and were interspersed among Schwann cells and astrocytes in the zone of neuroregeneration and were not observed in the adjacent white matter of the LES rats.

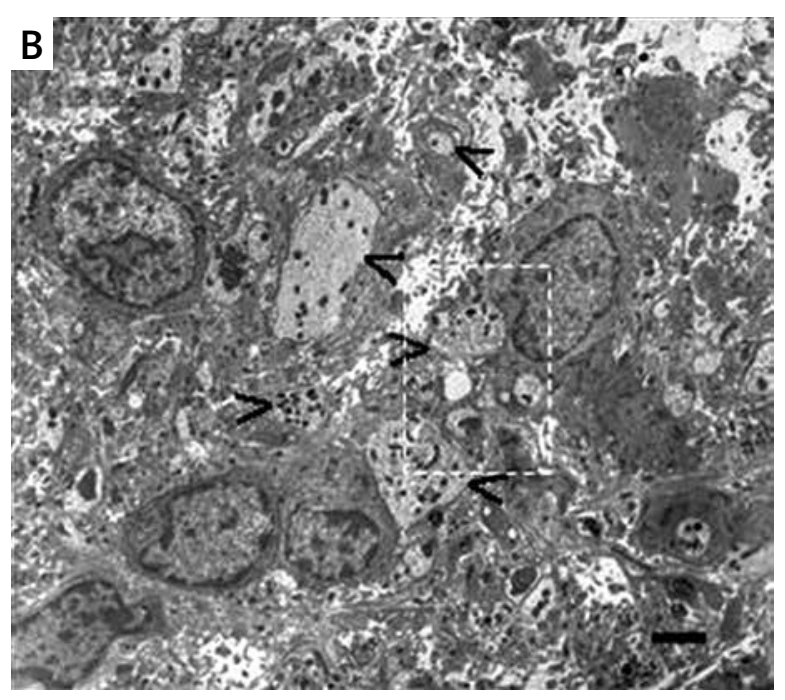

Fig. 6. Dorsal spinal column crush in the adult LES rat implanted with rat choroid plexus, 28 days post-op. (A) The crush cavity (Cc) contains clear fluid, presumably the CSF. At the interface of the crush cavity and the surrounding spinal cord tissue ( $\mathrm{Sc}$ ) there is a discontinuous layer of a zone of neuroregeneration (ZNR) delineated by a narrow cleft (open arrowheads) from the surrounding spinal cord tissue. TB, bar $50 \mu \mathrm{m}$. (B) TEM of the zone of neuroregeneration encompassing scattered cells forming microvilli and encasing axons (open arrowheads). Bar $5 \mu \mathrm{m}$. (C) A large cell (Ep) has abundant cytoplasm rich in organelles, forms numerous microvilli, encases a number of adjacent axons ( $\mathrm{x}$ ) with its cytoplasm and is interpreted as an ependymal cell. Bar $2 \mu \mathrm{m}$.

\section{Discussion}

The dysmyelinated CNS of the mature LES rats offers a unique in vivo model to study mechanisms of neuroregeneration and in this investigation, the site of the dorsal spinal cord crush injury implanted with rat choroid plexus was analyzed morphologically. The white matter injury in LES rats results in a limited and brief phagocytic inflammatory response 

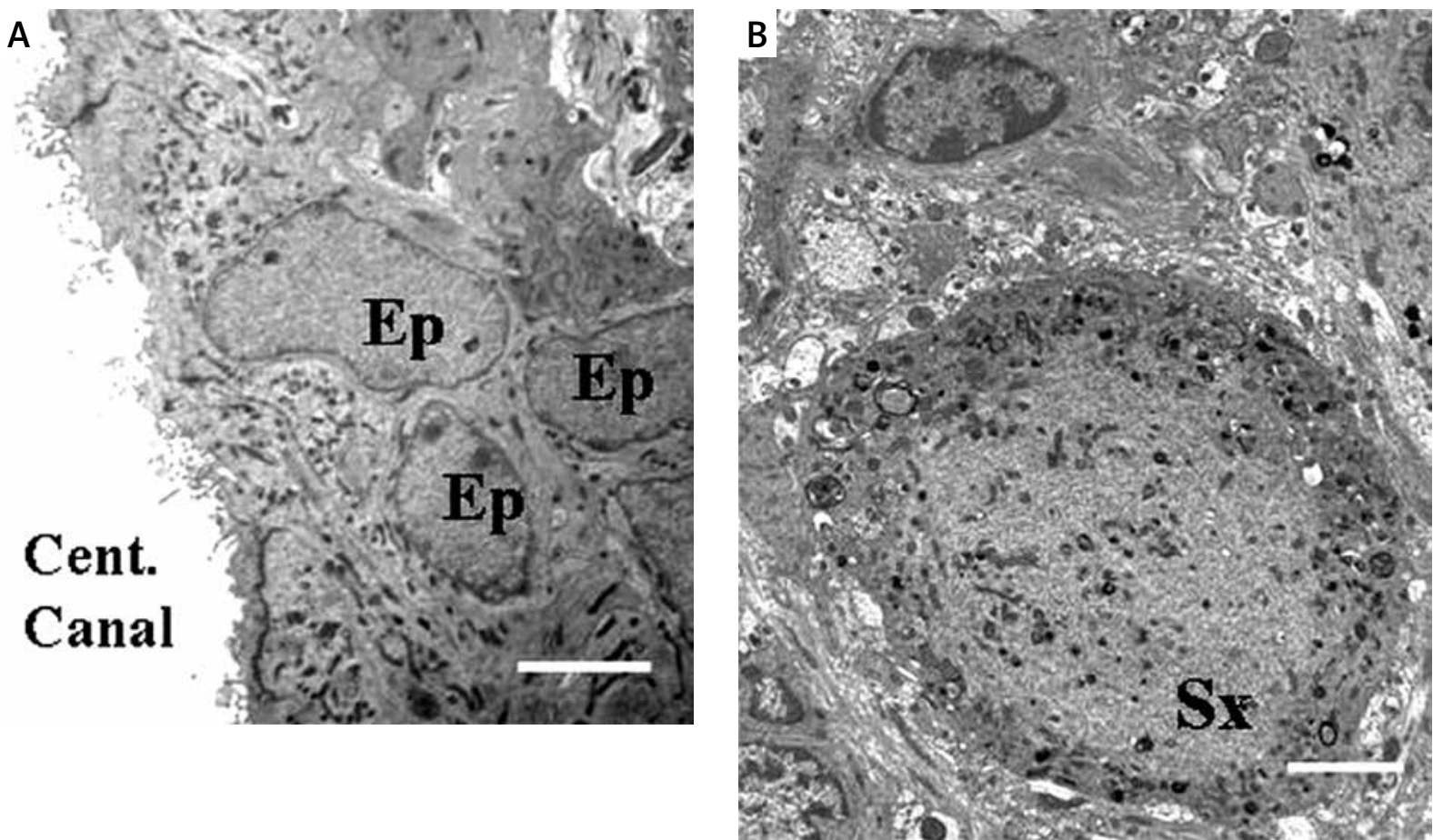

Fig. 7. Neural tissue surrounding the crash cavity of the LES rat, 28 days post-op. (A) The lumen of the central canal (Cent. Canal) is distended surrounded by hyperplastic ependymal cells (Ep) often forming two layers of cells. Bar $5 \mu \mathrm{m}$. (B) In the spinal cord tissue surrounding the crush cavity there are scattered swollen axons (Sx) filled with numerous mitochondria and vesicles in the sub-axolemma and packed neurofilaments and neurotubules in the centre of the axon. Bar $5 \mu \mathrm{m}$.

that clears before the day 7 post-op, therefore allowing for an unencumbered morphologic analysis of cellular mechanisms participating in neuroregeneration including axonal regeneration. In the crush cavity, the association of regenerating axons with ependymal cells from implanted choroid plexus was obligatory. A proportion of severed axons that were not encased by implanted cells and severed axons in crushed LES rats with no implant did not cross into the crush cavity.

\section{Inflammation}

The inflammatory response to the spinal cord injury in the mature myelinated CNS is severe, protracted and results in expansive tissue destruction [5]. Histological analysis has demonstrated macrophage-rich, severe infiltration of the injured tissue where macrophages were laden with lipid, myelinlike material suggesting that severity of the phagocytic response was directed against myelin damaged by injury and then by cytotoxic action of inflammatory cells [14] resulting in the mechanism of the vicious cycle where CNS tissue was progressively destroyed. Lack of any protracted and destructive, inflammatory response to the crush injury in the dysmyelinated spinal cord of the LES rats was likely related to the lack of potent pro-inflammatory stimulation by damaged myelin. This useful feature allowed for studying of cellular events following the crush and its implantation with rat choroid plexus including the inflammatory response against the implanted xenogeneic tissue. At day 3 post-op, numerous macrophages infiltrated the crush cavity and were interspersed with red blood cells, cellular debris and sheets of the epithelium from implanted choroid plexus which were consistent with an inflammatory response to the traumatic soft tissue injury. Similar pathology except the epithelial cells and fragments of blood vessels from choroid plexus stroma, was observed in the crush lesion of not implanted rats. Remarkably, prior to day 7 post-op, virtually all macrophages, tissue debris and red blood cells were removed from the crush cavity which in implanted rats contained almost exclusively dispersed ependymal cells encasing adjacent axons. The speed of clearance of inflam- 

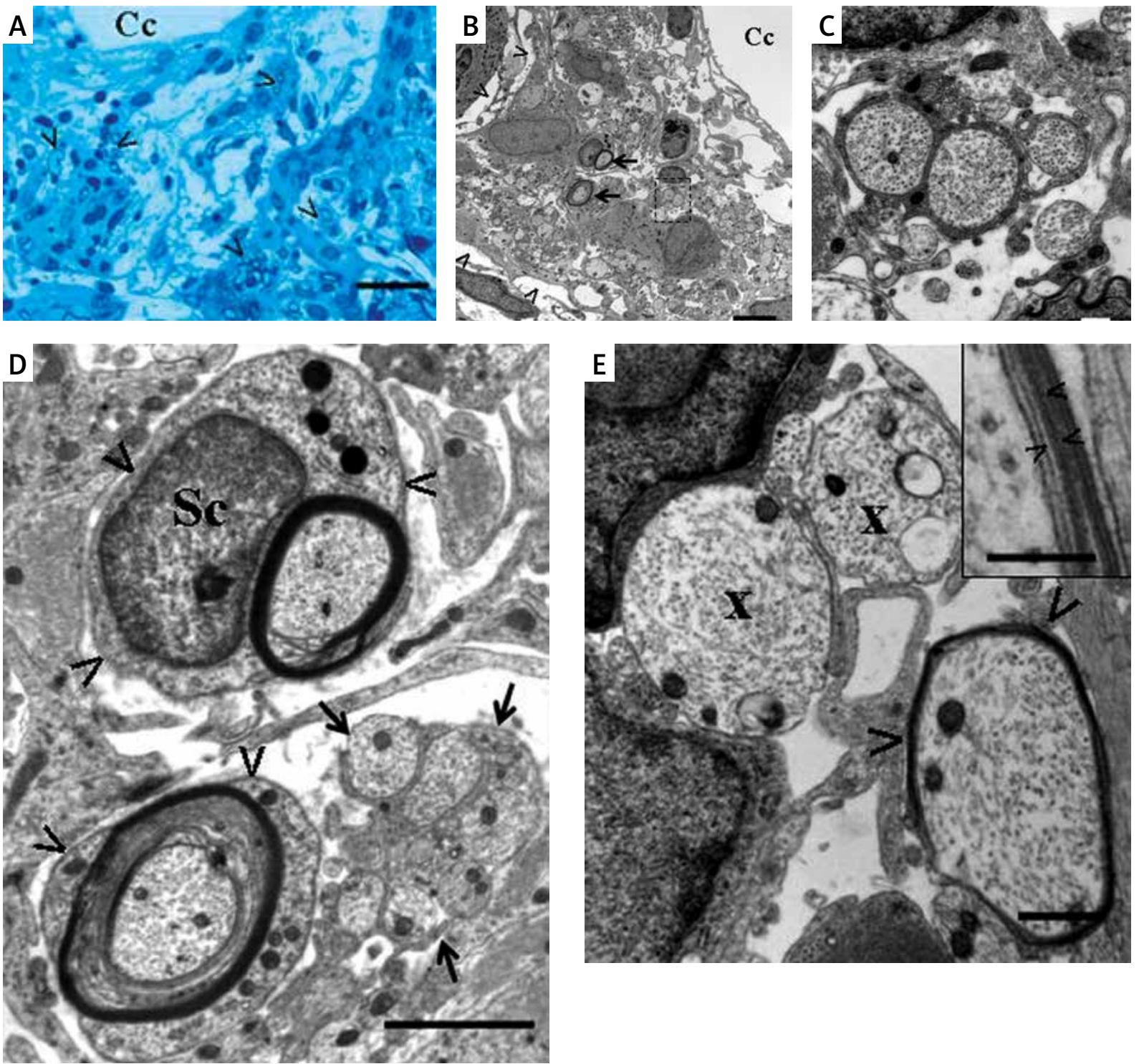

Fig. 8. Dorsal spinal column crush in the adult LES rat implanted with rat choroid plexus, 56 days post-op. (A) In the zone of neuroregeneration at the interface of the crush cavity (Cc) and surrounding spinal cord tissue, individual and small groups of cells are separated from each other by clear spaces interpreted as the CSF. TB, bar $50 \mu \mathrm{m}$. (B) TEM of the zone of neuroregeneration the crush cavity (CC) with scattered cells and cross sections of axons, two of which are myelinated. Bar $4 \mu \mathrm{m}$. (C) Higher magnification of an area from (B) in the box, a number of unmyelinated axons are encased by cytoplasmic processes of adjacent cells. Bar $500 \mathrm{~nm}$. (D) Higher magnification of another area indicated by two arrows in (B), there are two myelin sheaths of the Schwann cell type, with basal lamina, one adjacent to a Schwann cell, adjacent to a cluster of tightly adjacent, unmyelinated axons (arrows). Bar $2 \mu \mathrm{m}$. (E) In another area of the zone of neuroregeneration, there is a cell with a large, subcleaved nucleus and clumps of chromatin in periphery. Its cytoplasm is abundant, rich in ribosomes, endoplasmic reticulum, scattered mitochondria, apparatuses of Golgi and microtubules. This cell is interpreted as an oligodendrocyte, it has a cytoplasmic process encasing a large adjacent axon (arrowheads) that is surrounded by thin compacted myelin sheath. Two axons of similar diameter immediately adjacent to the cell $(\mathrm{x})$ are not myelinated. In the insert there is higher magnification of the myelin sheath from the axon (arrowheads) in (E) that forms major dense lines (arrowheads). Bar $1 \mu \mathrm{m}$, insert bar $200 \mathrm{~nm}$. 
matory infiltrate and cellular debris from the crush site in LES rats' spinal cord is considered unusually rapid for a soft tissue injury. The nature of an anti-inflammatory process leading to the rapid clearance of the phagocytic inflammatory response in the crush cavity is unknown but it may be related to soluble factors from the tissue surrounding the crush injury. It needs to be pointed however, that scattered macrophages laden with phagocytized red blood cells were observed in the areas of the CNS tissue surrounding the crush cavity for the entire duration (56 days) of the study (not shown).

\section{Axonal regeneration}

In the crush cavity implanted with the choroid plexus, numerous axons were encased by the ependymal cells. The association of axonal regeneration with implanted cells was obligatory in the crush cavity. Cut axons that did not enter the crush cavity were not encased by implanted cells in implanted rats and in crushed but not implanted rats. This close relationship of implanted cells and regenerating axons persisted throughout 2 months and apparently resulted in formation of new CNS tissue with the complement of axons, oligodendrocytes, astrocytes and Schwann cells in the zone of neuroregeneration at the interface of the crush cavity and the surrounding CNS tissue. Although this study focuses on the analysis of the site of the crush injury, it also indirectly addresses the following questions: (1) did axons regenerate past the site of injury? and (2) did regenerating ascending axons reach the original targets, approximately $5 \mathrm{~cm}$ distant at the nuclei cuneatus and gracilis in the caudal brain stem? The answer to the first question is affirmative and confocal microscopy analysis of traced regenerating axons is being performed in a currently ongoing study [14]. The answer to the second question is more circumstantial. In the developing nervous system only fully mature axons receive myelin sheaths [19], which implies that myelination of regenerating axons in this study was not observed in first 4 weeks but only at 8 weeks post-op because these axons took longer than 4 weeks to fully regenerate. The presence of numerous large diameter unmyelinated axons in the zone of neuroregeneration at 8 weeks post-op may suggest that a large proportion of regenerating axons did not mature fully to receive myelin and that there may be differences in the speed of re-growth among ascending axons in the dorsal column after their transection.

The ongoing tracing study will serve to shed more light on the mechanisms of axonal regeneration but clearly future in vivo studies on the LES rats will contribute a wealth of additional knowledge on cellular and molecular mechanisms regulating neuroregeneration leading to the cure of the CNS injury. The most important advantage from the present study lies in identifying the LES rat as the animal model where axonal regeneration can be conveniently manipulated and studied.

Numerous cut axons did not cross the implanted crush cavity and formed large bulb-like swellings packed with whirling neurofilaments and neurotubules, mitochondria and small dark vesicles which is similar to reported observations from previous studies on mature normally myelinated animal models including the cat [3], the rat [21], and in human cases studied ultrastructurally [23]. Axonal swellings were not encased by cell processes in this study and it is considered that these axons did not establish a connection with ependymal cells of the implanted choroid plexus and were unable to cross a fluid-filled crush cavity which was also shown previously [16]. It is unknown at the present what the differences between the ascending axons that did make contact with implanted ependymal cells and crossed the crush cavity and the axons that did not are. Perhaps enhancing the numbers of surviving implanted cells for a period of time sufficient for most of the cut axons to reach them and thus cross the lesion cavity would abrogate the need to address this question and thus address the optimization of neurologic recovery in the LES crush model. In any case, swollen axons persisted in the CNS tissue at the margin of the crush cavity in this study beyond 2 months postop. Studies addressing the ability of chronically transected axons to cross the crush cavity are planned for the near future in LES rats to address axonal regeneration in the chronic spinal cord injury.

An important question of the inhibitory effect of astrogliosis on axonal regeneration is directly addressed in this in vivo study and in the currently ongoing axonal tracing study [14]. Although astrogliosis in the intact CNS of the mature LES rats is very severe, it widely separates virtually every axon from each other $[10,11,13]$, it does not prevent the intact axons from retaining their plasticity evidenced as sprouting $[10,11,20]$. It also did not prevent axons 
from crossing the crush cavity when supported by implanted cells in this study and elongating in the dorsal column towards the brain stem targets in the currently ongoing axonal tracing study [14]. This in vivo evidence indicates that astrogliosis is not likely an important inhibitory mechanism for regenerating axons.

\section{Implanted cells}

The analysis of the crush site at days 3 and 7 postop indicates that a large proportion of the implanted choroid plexus tissue was eliminated from the crush injury together with extravascular red blood cells and other cellular debris from the crushed dorsal column. The presence of numerous macrophages in the crush lesion at day 3 post-op may suggest vigorous phagocytosis as the mechanism of removal of the red blood cells and cellular debris. Because at the time of the surgery the amount of the implanted choroid plexus filled the crush injury, it is considered that on the one hand a relatively small proportion of ependymal cells of the implanted choroid plexus survived the period of a few days pot-op and on the other, all surviving cells became dissociated from each other and all encased numerous axons at day 7 post-op and beyond. This observation points to a positive feedback mechanism where implanted cells survived when contacted by regenerating axons while cells not contacted by axons died off, perhaps in a process of apoptosis. If this hypothesis is true, enhancement of survival of implanted cells in the crush injury should increase the numbers of regenerating axons across the crush cavity with improved prognosis for neurological recovery. Of the cellular tissue components of the implanted choroid plexus only ependymal cells survived past day 7 post-op, presumably by being able to interact with regenerating axons and thus establish a vital connection with the tissue surrounding the crush cavity. Choroid plexus blood vessels and stromal cells were eliminated from the crush cavity by day 7 post-op.

Changes in the distribution of the implanted choroid plexus epithelial cells in the crush cavity appear to follow a highly dynamic pathway suggestive of strong interactions among the implanted cells, the regenerating axons and the CNS tissue surrounding the crush cavity. At day 3 post-op, individual cells within small sheets of columnar epithelium were encasing regenerating axons. At day 7 post-op, instead of sheets of epithelial cells there were dissociated large cells encasing numerous axons that were similar to immature ependymal cells [11] and were dispersed over the crush cavity. This distribution pattern changed dramatically prior to day 14 postop when implanted cells with encased axons clustered exclusively in the zone of neuroregeneration at the interface of the crush cavity and the surrounding CNS tissue. It should be pointed that cross sections of the cells or groups of cells encasing axons should be considered as cross sections of elongated structures where rows of implanted cells span the width of the crush cavity serving as a functional bridge for axonal crossing of the injury site. Observations of migration of ependymal cells towards the site of injury made in previous studies $[4,17]$ support the notion that these cells are highly and rapidly responsive to a CNS tissue injury. In this study the implanted cells and regenerating axons persisted in the zone of neuroregeneration thus forming new tissue that at 56 days post-op included axons, a proportion of which were myelinated, with a complement of Schwann cells, oligodendrocytes and astrocytes. In a previous study on a spinal cord injury rat model, implanted choroid plexus ependymal cells associated with transected axons and supported their limited regeneration for at least 10 months [6].

Formation of myelin around a proportion of axons in the zone of neuroregeneration observed at day 56 post-op has two fundamental implications. Firstly, the formation of myelin sheaths complete with a major dense line by oligodendrocyte-like cells indicates that implanted choroid plexus ependymal cells trans-differentiated into oligodendrocytes that formed thin but properly compacted myelin sheaths. Rare thin sheaths that intact LES rat oligodendrocytes form at young age are uncompacted and lacking a major dense line [13], whereas sheaths are not formed spontaneously in LES rats at older ages [10] that were used for the present study. This observation further confirms that the epithelium of the choroid plexus is the source of neural progenitor cells $[7,9]$, some of which can give rise to oligodendrocytes. Secondly, myelination of a proportion of axons in the zone of neuroregeneration may be suggestive that these axons attained maturity and re-grew fully [19] prior to day 56 post-op. Both cellular mechanisms of CNS regeneration offer exciting possibilities and will need to be addressed in specific in vivo studies. 
The most important objective of neurorestoration is full anatomical axonal regeneration, however, it has been elusive in in vivo models thus far. The LES rat model where the axonal regeneration and involved cellular mechanisms can be conveniently studied will likely contribute to our understanding of how cut CNS axons regenerate.

\section{Acknowledgments}

Funding support for this project was provided by the Department of Surgery and the Faculty of Health Science, McMaster University.

\section{References}

1. Chakrabortty S, Kitada M, Matsumoto N, Taketomi M, Kimura K, Ide C. Choroid plexus ependymal cells enhance neurite outgrowth from dorsal root ganglion neurons in vitro. J Neurocytol 2000; 29: 707-717.

2. Emerich DF, Skinner SJM, Borlongan CV, Vasconcellos AV, Thanos CG. The choroid plexus in the rise, fall and repair of the brain. BioEssays 2005; 27: 262-274.

3. Erb DE, Povlishok JT. Axonal damage in severe traumatic brain injury: an experimental study in cat. Acta Neuropathol 1998; 76: 347-358.

4. Horky LL, Galimi F, Gage FH, Horner PJ. Fate of endogenous stem/progenitor cells following spinal cord injury. J Comp Neurol 2006; 498: 525-538.

5. Hughes JT. Disorders of the spine and spinal cord. In: Adams JH, Duchen LW (eds.). Greenfield's Neuropathology. $5^{\text {th }}$ ed. Oxford University Press, New York 1992, pp. 1083-1115.

6. Ide C, Kitada M, Chakrabortty S, Taketomi M, Matsumoto N, Kikukawa S, Mizoguchi A, Kawaguchi S, Endoh K, Suzuki Y Grafting of choroid plexus ependymal cells promotes the growth of regenerating axons in the dorsal funiculus of rat spinal cord: A preliminary report. Exp Neurol 2001; 167: 242-251.

7. Itokazu Y, Kitada M, Dezawa M, Mizoguchi A, Matsumoto N, Shimizu, Ide C. Choroid plexus ependymal cells host neural progenitor cells in the rat. Glia 2006; 53: 32-42.

8. Johansson CB, Momma S, Clarke DL, Risling M, Lendahl U, Frisen J. Identification of a neural stem cells in the adult mammalian central nervous system. Cell 1999; 96: 25-34.

9. Kitada M, Chakrabortty S, Matsumoto N, Taketomi M, Ide C. Differentiation of choroid plexus ependymal cells into astrocytes after grafting into the pre-lesioned spinal cord in mice. Glia 2001; 36: 364-374.

10. Kwiecien JM. Cellular compensatory mechanisms in the CNS of dysmyelinated rats. Comp Med 2010; 60: 205-217.

11. Kwiecien JM, Avram R. Long distance axonal regeneration in the filum terminale of adult rats is regulated by ependymal cells. J Neurotrauma 2008; 25: 196-204.

12. Kwiecien JM, Delaney KH. Endpoints in myelin deficient (md) rat: Acute hemorrhagic myelitis attributed to spinal hyperflexion and vertebral fracture. Comp Med 2010; 60: 343-347.
13. Kwiecien JM, O'Connor LT, Goetz BD, Delaney KH, Fletch AL, Duncan ID. Morphological and morphometric studies of the dysmyelinating mutant, the Long Evans shaker rat. J Neurocytol 1998; 27: 581-591.

14. Kwiecien JM, Rukholm G, Khetani J, MacLean J, Avram R, Robertson DD, Archibald J, Lach B. Neuroregeneration in the acute spinal cord injury in adult dysmyelinated rat implanted with choroid plexus. Abstract presented at the International Congress of Neuropathology, Salzburg, Austria, September, 2010. Brain Pathol 20(S1): 1-99.

15. Li Y, Chen J, Chopp M. Cell proliferation and differentiation from ependymal, subependymal and choroid plexus cells in response to stroke in rats. J Neurol Sci 2002; 193: 137-146.

16. MCPhail LT, Stirling DP, Tetzlaff W, Kwiecien JM, Ramer MS. The contribution of activated phagocytes and myelin degeneration to axonal retraction/dieback following spinal cord injury. Eur J Neurosci 2004; 20: 1984-1994.

17. Mothe AJ, Tator CH. Proliferation, migration, and differentiation of endogenous ependymal region stem/progenitor cells following minimal spinal cord injury in the adult rat. Neurosci 2005; 131: 177-187.

18. O'Connor LT, Goetz BD, Kwiecien JM, Delaney KH, Fletch AL, Duncan ID. Insertion of a retrotransposon into the myelin basic protein gene causes CNS dysmyelination in the Long Evans shaker (LES) rat. J Neurosci 1999; 19: 3404-2413.

19. Peters A, Palay SL, Webster Hde E (eds.). The Fine Structure of the Nervous System. WB Saunders Company, Toronto 1976.

20. Phokeo V, Kwiecien JM, Ball AK. Characterization of the optic nerve and retinal ganglion cell layer in the dysmyelinated adult Long Evans Shaker rat: possible axonal sprouting. I Comp Neurol 2002; 451: 213-224.

21. Reeves TM, Phillips LL, Povlishok JT. Myelinated and unmyelinated axons of the corpus callosum differ in vulnerability and functional recovery following traumatic brain injury. Exp Neurol 2005; 196: 126-137.

22. Schwab JM, Brechtel K, Mueller CA, Failli V, Kaps HP, Tuli SK, Schluesener HJ. Experimental strategies to promote spinal cord regeneration - an integrative perspective. Prog Neurobiol 2006; 78: 91-116

23. Smith PM, Jeffery ND. Histological and ultrastructural analysis of white matter damage after naturally-occurring spinal cord injury. Brain Pathol 2006; 16: 99-109.

24. Takahashi M, Arai Y, Kurosawa H, Sueyoshi N, Shirai S. Ependymal cell reactions in spinal cord segments after compression injury in adult rat. J Neuropathol Exp Neurol 2003; 62: 185-194.

25. Xu X-M, Onifer SM. Transplantation-mediated strategies to promote axonal regeneration following spinal cord injury. Resp Physiol Neurobiol 2009; 169: 171-182.

26. Xu Y, Tamamaki N, Noda T, Kimura K, Itokazu Y, Matsumoto N, DezawaM, IdeC. Neurogenesisintheependymallayer oftheadult rat $3^{\text {rd }}$ ventricle. Exp Neurol 2005; 192: 251-264.

27. Zhang RL, Zhang ZG, Wang Y, LeTourneau Y, Liu XS, Zhang X, Gregg SR, Wang L, Chopp M. Stroke induces ependymal cell transformation into radial glia in the subventricular zone of the adult rodent brain. J Cereb Blood Flow Metab 2007; 27: 1201-1212. 